\title{
Atomic-scale perspective on the origin of attractive step interactions on $\operatorname{Si}(113)$
}

\author{
Cristian V. Ciobanu, Dhananjay T. Tambe and Vivek B. Shenoy \\ Division of Engineering, Brown University, Providence, RI 02912 \\ Cai-Zhuang Wang and Kai-Ming Ho \\ Ames Laboratory - USDOE and Department of Physics, Iowa State University, Ames IA 50011
}

(Dated: November 2, 2018)

\begin{abstract}
Recent experiments have shown that steps on $\mathrm{Si}(113)$ surfaces self-organize into bunches due to a competition between long-range repulsive and short-range attractive interactions. Using empirical and tight-binding interatomic potentials, we investigate the physical origin of the short-range attraction, and report the formation and interaction energies of steps. We find that the short-range attraction between steps is due to the annihilation of force monopoles at their edges as they combine to form bunches. Our results for the strengths of the attractive interactions are consistent with the values determined from experimental studies on kinetics of faceting.
\end{abstract}

Self-assembly of steps is an important way to achieve surface patterning at length scales where the usual lithographic techniques are not applicable. On certain vicinal semiconductor surfaces, the equilibrium bunching of steps leads to the formation of periodic hill-and-valley patterns (or grooves), that can serve as templates for growing regular arrays of one-dimensional (1D) nanoscale devices like quantum wires. Experimental work in recent years [1, 2, 3, 4, 5] has revealed that such groove structures are formed on vicinal $\mathrm{Si}(113)$, where the flat (113) surface coexists with step-bunched regions over a wide range of temperatures and misorientation angles.

In a series of experiments, Mochrie and coworkers [1, 2, 3] have studied the orientational phase diagrams for stepped $\mathrm{Si}(113)$ surfaces miscut towards several crystallographic orientations. In all the cases, their studies strongly suggest that, at low temperatures, the steps gather in bunches as a result of attractive step-step interactions. Independent experimental work on the fluctuations of the single and multiple-height steps and the kinetics of faceting transitions by Sudoh and coworkers [4, 5] have confirmed the presence of short-range attractive interactions between steps. Furthermore, their analysis of the step-zipping process has provided quantitative estimates for the magnitude of the attractive interactions 5]. The presence of such interactions has been assumed in theoretical models that show the orientational phase diagram [1] can be explained in terms of competing short-range attractive and long-range repulsive step interactions 6,6 ]. While much progress has been made in understanding the self assembly of steps on $\mathrm{Si}(113)$ surfaces, a microscopic analysis of the step interactions has not been attempted and the physical origin of the shortrange attraction has not been convincingly explained.

In this letter, we investigate the interactions between steps on $\mathrm{Si}(113)$, with the goal of understanding the origin of the effective short-range attraction between steps. Describing each step as a pair of equal and opposite force monopoles located at the step edges, we find that the short-range attraction between steps is due to the can- cellation of force monopoles that belong to adjacent steps in a step bunch. This observation is confirmed by investigating the scaling of the step formation energies and interaction strengths with the step height. Noting that the structure of the steps is determined by the direction of the miscut, we consider here $\mathrm{Si}(113)$ surfaces miscut towards [332], so that our estimates for the magnitudes of step interactions can be directly compared with the recent experiments of Sudoh et al. [5].

The slab geometry that shows the $\mathrm{Si}(113)$ terraces and types of steps studied in the present work is given in

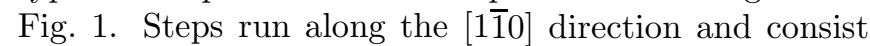
of (114) nanofacets [5], with a $(2 \times 1)$ reconstruction [8]. For the $(113)$ surface we consider both the $(3 \times 1)$ and the $(3 \times 2)$ structures $[9]$ that are stabilized by interstitial atoms. We distinguish two possible structures for the $(3 \times 2)$ case, depending on the position of the interstitial rows on the terraces with respect to the step. When the interstitials on the lower terrace are away from the step or close to the step, we call them the $(3 \times 2)$ a and the $(3 \times 2)$ c structures, respectively (refer to Fig. 11).

The dimension of the simulation cell in the $x$-direction, $L_{x}$, is determined by the number $k$ of $\mathrm{Si}(113)$ unit cells and the number $n$ of $\mathrm{Si}(114)$ unit cells that constitute the step: $L_{x}=n \Delta+k a_{x}$, where $a_{x}=12.73 \AA$ and $\Delta=$ $16.21 \AA$ are, respectively, the periodic lengths of the (113) and the (114) surfaces along [332] (Fig. 11). The periodic length in the $[1 \overline{1} 0]$ direction $L_{y}$ is the smallest length compatible with the periodicity of both the $\mathrm{Si}(113)$ and the $\operatorname{Si}(114)$ structures, $L_{y}=23.04 \AA$. The thickness of the slab $L_{z}$ was chosen to be $\sim 150 \AA$. To simulate the vicinal geometry, we use shifted periodic boundary conditions [10] in which the shift along [113] is determined by the step height $n h$, where $h=1.64 \AA$ is the height of a single step.

The energies of the slabs in Fig. 1 are computed using empirical and tight-binding (TB) models for the atomic interactions 11]. Empirical calculations are carried out with the Tersoff potential T2 [12] as it reproduces the structure, surface stress and relative energy of the $\mathrm{Si}(113)$ 


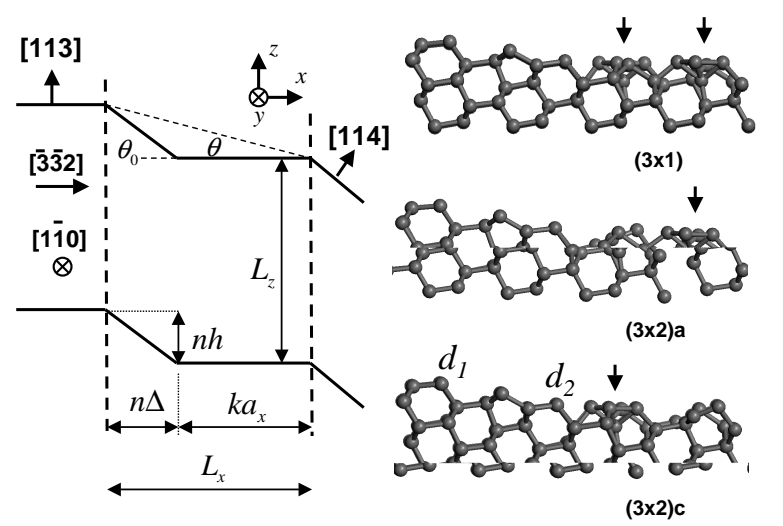

FIG. 1: (a) Geometry of a typical stepped Si(113) surface with the simulation cell enclosed between thick dashed lines. Different step structures with $n=k=1$ (miscut $\theta=3.2^{\circ}$ ) are shown on the right. Arrows mark the positions of the interstitial atoms that stabilize the terraces [9]. For the $(3 \times 2)$ surfaces, there are two possible ways of creating a step: the interstitial rows on the lower terrace can be located either away from the step, $(3 \times 2)$ a, or close to the step, $(3 \times 2)$ c. The symbols $d_{1}$ and $d_{2}$ label the dimers on the (114) step.

and $\operatorname{Si}(114)$ reconstructions reasonably well. While the tight-binding methods are more accurate, they are usually impractical for ledge energy calculations since they require slabs with several thousands of atoms to capture the long-range interactions between steps. To circumvent this problem, we have developed a multi-scale approach where the tight-binding model is coupled to an empirical potential as described in the following paragraph.

The key idea of the coupling scheme is to handle the long-range elastic fields produced by steps using an empirical potential whose elastic constants are very close to their TB counterparts; this would ensure that the elastic fields in the hybrid scheme would not be very different from the fields obtained in the case where all the atoms were treated using TB. We use a charge selfconsistent TB method, whose superior transferability has been studied in detail in [13]. This method is coupled with the Tersoff (T3) potential 14 using the scheme shown in Fig. 2 We divide the simulation cell into three regions: (1) a thin surface region where the complex bonding topologies in the vicinity of the step-edge are treated with the TB method, (2) a thick "bulk-like" region where all the atoms with tetragonal bonding environments are treated with the T3 method and (3) a padding zone which is used to communicate atomic displacements between the first two regions. The atoms in the simulation cell are relaxed using the following procedure (refer to Fig. 2): (1) relax the thin slab (Zone 1, Fig. 2(a)) using TB, while keeping the padding zone fixed; (2) update the atomic coordinates of Zone 1 in Fig. 2(b) with the values obtained after the TB relaxation of Zone 1 in Fig. 2(a); (3) relax the thick slab and

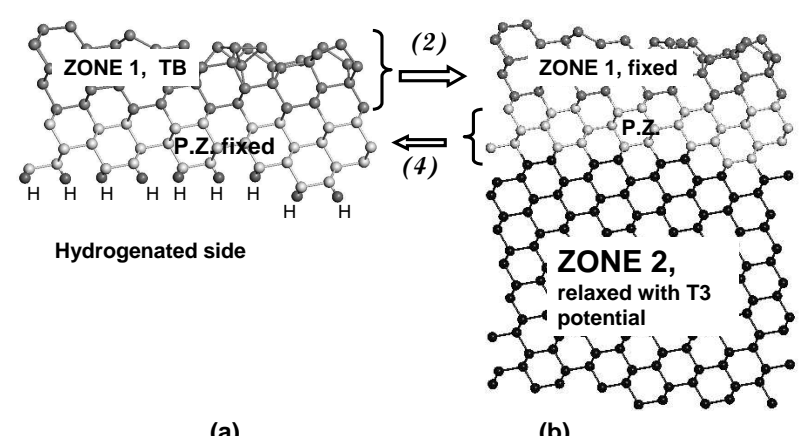

(b)

FIG. 2: Relaxation scheme employed to compute ledge energies using a coupling of the tight-binding (TB) method [13] with the T3 potential [14]. Zone 1 (thin) consists of a few surface layers that are relaxed using TB (a), but are kept fixed during the relaxation of the thick slab (b). Zone 2 (thick) represents the deeper layers, which interact with the surface layers through a padding zone P.Z. The arrows show how atomic coordinates are updated during stages (2) and (4) of the procedure described in text.

the padding zone (Zone 2 and P.Z. in Fig. 2(b)) using the T3 potential while keeping the atoms in Zone 1 fixed; (4) update the coordinates of the P.Z. atoms in Fig. 2(a) with the values obtained after the T3 relaxation of P.Z. in Fig. 2(b); (5) repeat steps (1) through (4) until energy convergence is achieved 15.

Once the total energy $E$ of the slab is calculated, step interactions can be obtained from the ledge energy [10]

$$
\lambda\left(L_{x}\right) \equiv\left(E-N e_{b}-\gamma_{113} L_{x} L_{y}\right) / L_{y},
$$

where $e_{b}$ is the bulk cohesion energy per atom [16], $N$ is the number of atoms in the cell in Fig. [1 and $\gamma_{113}$ is the surface energy of $\operatorname{Si}(113)$. Using the fact that the steps interact through long-range dipolar fields [17], we have analyzed the short-range interactions by fitting the ledge energies in Eq. (11) to the form []

$$
\lambda\left(L_{x}=n \Delta+k a_{x}\right)=\Lambda_{n}+G_{n} \frac{\pi^{2} \Delta^{2}}{6 L_{x}^{2}}-U_{n} \delta_{k 0},
$$

where $\Lambda_{n}$ is the formation energy per unit length of a step of height $n h, G_{n}$ is the strength of the long-range dipolar interactions, and $\delta_{k 0}$ denotes the Kronecker delta symbol, where $k$ is the number of (113) unit cells on the terrace (Fig. 1). The energy offset $U_{n}$ that appears in Eq. (2) describes the effective interactions at the smallest possible spacing between the steps, $n \Delta$ (note that the (113) terraces are absent in this case). A positive (negative) value of $U_{n}$ indicates the presence of a short-range attraction (repulsion) between steps. Our results for different terrace and step structures are discussed below.

We first focus attention on the vicinal surfaces made of single-height steps and illustrate the general features of the step interactions by considering the energetics of the 


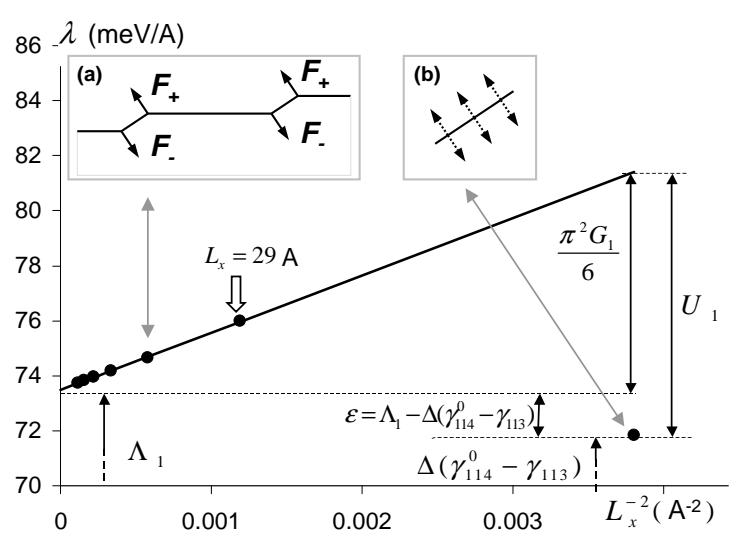

FIG. 3: Ledge energy $\lambda$ of the single-height (114) steps separated by $(3 \times 2)$ c terraces, plotted as a function of $1 / L_{x}^{2}$. The points corresponding to separations $L_{x}>\Delta=16.21 \AA$ are fit to a straight line, $\lambda=\Lambda_{1}+\pi^{2} G_{1} \Delta^{2} / 6 L_{x}^{2}$. At the smallest separation $\Delta$, the ledge energy falls below the straight line, indicating a short-range attraction $U_{1}=\pi^{2} G_{1} / 6+\Lambda_{1}-\Delta\left(\gamma_{114}^{0}-\right.$ $\gamma_{113}$ ) [18]. The inset (a) shows an array of steps (simulated via periodic boundary conditions) where each step is represented schematically as a pair of force monopoles $F_{+}$and $F_{-}$. When $L_{x}=\Delta$, there are no terraces between steps and the monopoles corresponding to adjacent steps cancel each other, as shown in inset (b).

$(3 \times 2)$ c steps obtained from the T2 potential. In Fig. 3 we plot the ledge energy as function $1 / L_{x}^{2}$ ( $L_{x}$ is the step separation) and provide the fitting parameters $\Lambda_{1}, G_{1}$ and $U_{1}$ defined in Eq. (2). If the dipolar description of step interactions is assumed to hold over the entire range of step separations (as implied by Eq. (2)), Fig. 31 shows the presence of a short-range attraction $\left(U_{1}\right)$, since the point corresponding to $L_{x}=\Delta$ lies below the linear fit for the ledge energy. The physical reason for the origin of this attraction will be discussed next.

We start by noting that the $\mathrm{Si}(113)$ and $\mathrm{Si}(114)$ facets are atomically compatible, in the sense that they can meet to form edges parallel to [11̄0] without any changes in their individual bonding topologies. Subsequently, the elastic fields of a (114) step (or nanofacet) can be expressed in terms of a dipole which consists of a pair of equal and opposite force monopoles $\left(F_{+}, F_{-}\right)[19$ at the edges of the step, as shown in inset (a) of Fig. [3] While such steps are expected to show inverse-square repulsive interactions at large separations, we find that this behavior holds for separations as small as $29 \AA$ (this point is marked in Fig. (3). When the steps are closer than $29 \AA$, they lose their individuality forming a smooth $\mathrm{Si}(114)$ surface: at a separation of $L_{x}=\Delta$ there is a cancellation of the monopoles at the step-edges as illustrated in inset (b) of Fig. 3 The absence of dipoles with mutual repulsive interactions is the reason for the presence of an effective short-range attraction between steps. Further evidence for this argument is obtained by computing the

\begin{tabular}{llccccc}
\hline \hline Method & Structure & $U_{1}$ & $G_{1}$ & $U_{1} / G_{1}$ & $\Lambda_{1}$ & $\varepsilon$ \\
\hline \multirow{3}{*}{ TB-T3 } & $(3 \times 1)$ in & 61.68 & 18.84 & 3.27 & 108.94 & 26.05 \\
& $(3 \times 1)$ out & 60.12 & 13.65 & 4.40 & 110.28 & 33.08 \\
& $(3 \times 2) c$, in & 62.6 & 8.69 & 7.2 & 66.48 & 45.20 \\
\hline \multirow{2}{*}{ T2 } & $(3 \times 1)$ & 8.74 & 10.45 & 0.84 & 173.59 & -8.44 \\
& $(3 \times 2) \mathrm{a}$ & 0.22 & 3.70 & 0.06 & 65.98 & -5.86 \\
& $(3 \times 2) \mathrm{c}$ & 9.30 & 4.81 & 1.93 & 73.48 & 1.64 \\
\hline Exp. [5] & $(3 \times 2)$ & & & & 57 & $13-15$ \\
\hline \hline
\end{tabular}

TABLE I: Interaction strengths and step formation energies for single-height steps computed using the multiscale tightbinding/Tersoff scheme (TB-T3) and the T2 potential [12]. The labels "in" and "out" denote the relative tilting (in- and out-of-phase) of the dimer rows $d_{1}$ and $d_{2}$ shown in Fig. 1] The last column shows the quantity $\varepsilon \equiv \Lambda_{1}-\Delta\left(\gamma_{114}^{0}-\gamma_{113}\right)$, which allows for a direct comparison with the experimental results (Exp. 5]). All quantities (except the ratio $U_{1} / G_{1}$ ) are given in $\mathrm{meV} / \AA$.

interactions of multiple-height steps. We will focus on such calculations after we discuss the interaction parameters for other types of steps given in Fig. 1.

Using both the T2 potential and the TB-T3 method, we find the existence of short-range attractive interactions between steps for all the reconstructed surfaces considered here. However, the magnitudes of these interactions depend strongly on the structures of the terraces and steps (refer to Table 【). Since the TB method allows for the tilting of the dimers $d_{1}$ and $d_{2}$ on the step (marked in Fig. 1), we have reported the results for the cases where the tilting patterns are in-phase and outof-phase [20]. The magnitude of the attractive interaction $U_{1}$ is $\approx 60 \mathrm{meV} / \AA$ and $\approx 10 \mathrm{meV} / \AA$ for the TB-T3 method and $\mathrm{T} 2$ potential, respectively. This discrepancy between the values of $U_{1}$ computed with TB-T3 and with $\mathrm{T} 2$ is due to the differences in step formation energies obtained with the two methods, as well as to the sensitivity of $U_{1}$ with respect to changes in $\left(\gamma_{114}^{0}-\gamma_{113}\right)$ (refer to Fig. 31). For both T2 and TB-T3, the repulsion strength $G_{1}$ is of the order of $10 \mathrm{meV} / \AA$, while the formation energy $\Lambda_{1}$ is of the order of $100 \mathrm{meV} / \AA$ (see Table 【). In order to compare our results with the experimental ones [5], we note that Sudoh et al. 5] report the quantity $\varepsilon \equiv \Lambda_{1}-\Delta\left(\gamma_{114}^{0}-\gamma_{113}\right)$, i.e. the difference between the step formation energy $\Lambda_{1}$ and the excess energy of the $\mathrm{Si}(114)$ facet per length of the step. Table \ shows that the values for $\varepsilon$ obtained with the TB-T3 method are of the same order of magnitude as the estimates of Sudoh et al. [5]. Closer agreement could be achieved if the longrange interactions are included in the analysis of kinetics of step-zipping reported in [5]. Also, future experiments aimed at determining the structure of the step ( $a$ or $c$ ) could verify the trends given in Table \

We will now focus on the formation energy and interactions of steps with height $n h$ ( $n$-bunches) for $n=2,3$. 


\begin{tabular}{ccccccc}
\hline \hline$n h$ & $\Lambda_{n}$ & $n \Lambda_{1}$ & $\Lambda_{n}$, Eq. (3) & $G_{n}$ & $n^{2} G_{1}$ & $U_{n}$ \\
\hline$h$ & 73.48 & 73.48 & & 4.81 & 4.81 & 9.3 \\
$2 h$ & 143.48 & 146.96 & 142.48 & 19.36 & 19.24 & 7.7 \\
$3 h$ & 213.62 & 220.44 & 212.67 & 47.88 & 43.29 & 7.0 \\
\hline \hline
\end{tabular}

TABLE II: Step-height dependence of the formation energy $\Lambda_{n}$ and interaction parameters $G_{n}, U_{n}$ for the $(3 \times 2)$ c structure, determined with the Tersoff potential T2 [12]. All quantities are given in $\mathrm{meV} / \AA$.

Because of the larger size of the simulation cells, we have used only the T2 potential 12 for this analysis. In the zero temperature limit, the formation energy of an $n$ bunch can be expressed as the sum of the formation and interaction energies of the steps within the bunch [7]:

$$
\Lambda_{n}=n \Lambda_{1}-U_{1}(n-1)+G_{1} \sum_{i=1}^{n-1} \frac{n-i}{i^{2}} .
$$

Due to the presence of attractive interactions between single-height steps, the formation energy of an $n$-height step is expected to show sub-linear dependence of $n$, $\Lambda_{n}<n \Lambda_{1}$ [7]. This prediction is indeed borne out by the atomistic results given in Table Using the parameters $\Lambda_{1}, G_{1}, U_{1}$ from Table $\llbracket$ for the $(3 \times 2)$ c structure, we find that the bunch energies predicted by Eq. (3) are within $1-2 \mathrm{meV} / \AA$ of the values for $\Lambda_{n}$ obtained in the present simulations.

Turning our attention to the step-step interactions, we find that the repulsive interactions $G_{n}$ show a nearly quadratic dependence of the step height $n h$, while the strength of the attractive interaction $U_{n}$ shows a weak dependence 21] on $n$ (refer to Table III). The quadratic behavior of $G_{n}$ provides strong evidence to the idea regarding the cancellation of the force monopoles associated with the adjacent steps in an $n$-bunch. When such cancellation takes place, the only remaining monopoles are associated with the ends of the bunch. Consequently, each $n$-bunch behaves as a force-dipole with a moment that is proportional to $n h$. Identical $n$-bunches interact as dipoles 17], with a repulsion strength determined by the square of the dipolar moment of one $n$-bunch, which results in the $n^{2}$ dependence of $G_{n}$.

In conclusion, we have shown that the effective shortrange attraction experienced by steps on $\mathrm{Si}(113)$ can be explained by the cancellation of force monopoles associated with adjacent steps in a step bunch. While numerical estimates for the interaction parameters depend on the interatomic potential used, our physical reasoning for the origin of attractive interaction is robust and is consistent with experiments. A key point in understanding how the atomic structure can lead to step-step attractive interactions is the atomic compatibility of the two stable surface orientations, $\operatorname{Si}(113)$ and $\operatorname{Si}(114)$ : when nanofacets with these orientations intersect forming a step edge, no bond breaking or rebonding occurs. Recent experimental work 22 indicates that no rebonding occurs at the step-edges formed between the $\mathrm{Si}(113)$ and $\mathrm{Si}(337)$ facets; our calculations show the presence of attractive interactions between steps in this case, further lending support to our physical picture. The difference between the type of steps considered here, and the most common steps on $\mathrm{Si}(001)$ surfaces, such as the $\mathrm{SB}$ and the DB steps [23] is that when steps on $\mathrm{Si}(113)$ merge they form a new facet (e.g. $\mathrm{Si}(114)$ ), while such atomically smooth facets are not observed in the latter case due to significant rebonding at the step-edges [23]. Therefore, a simple cancellation of force monopoles is not expected for the $\mathrm{SB}$ and the $\mathrm{DB}$ steps on $\mathrm{Si}(001)$, and hence they do not show attractive interactions 10. Further theoretical and experimental studies aimed at elucidating the dependence of the step-step interactions of the azimuthal orientation will shed more light on the self-assembly of steps on $\mathrm{Si}(113)$ miscut in an arbitrary direction.

Support from the MRSEC at Brown University (DMR0079964), NSF (CMS-0093714, CMS-0210095), and the Salomon Research Award from the Graduate School at Brown University is gratefully acknowledged. Ames Laboratory is operated by the U.S. Dept. of Energy and by the Iowa State University (contract W-7405-Eng-82).

[1] S. Song and S.G.J. Mochrie, Phys. Rev. Lett. 73, 995 (1994); Phys. Rev. B 51, 10068 (1995).

[2] S. Song et al., Surf. Sci. 372, 37 (1997).

[3] M. Yoon et al., Surf. Sci. 411, 70 (1998).

[4] K. Sudoh et al., Phys. Rev. Lett. 80, 5152 (1998); Surf. Sci. 419, 128 (1999).

[5] K. Sudoh et al., Surf. Sci. 452, L287 (2000); Phys. Rev. Lett. 87, 216103 (2001).

[6] S.M. Bhattacharjee, Phys. Rev. Lett. 76, 4568 (1996); M. Lässig, Phys. Rev. Lett. 77, 526 (1996).

[7] V.B. Shenoy et al.,Phys. Rev. Lett. 81, 3475 (1998); Surf. Sci. 467, 58 (2000).

[8] S.C. Erwin et al., Phys. Rev. Lett. 77, 687 (1996).

[9] J. Dąbrowski et al., Phys. Rev. Lett. 73, 1660 (1994); Surf. Sci. 331-333, 1022 (1995).

[10] T.W. Poon et al., Phys. Rev. B 45, 3521 (1992).

[11] Ab-initio studies to capture the elastic interactions between steps on the (113) surfaces would require prohibitive amounts of computational resources.

[12] J. Tersoff, Phys. Rev. B 37, 6991 (1988).

[13] C-Z. Wang, et al., J.Phys. Cond. Mat. 11, 2043 (1999); Surf. Sci. 436, L697 (1999).

[14] J. Tersoff, Phys. Rev. B 38, 9902 (1988). This potential (T3) was used in the coupling scheme because its elastic constants are close to the tight-binding values [13].

[15] Implementation details of this multiscale procedure will be reported elsewhere. A similar coupling scheme, though not self-consistent, was used by J. Wang et al. in Phys. Rev. B 47, 10497 (1993) to compute the formation energy of the dimer-vacancies on $\mathrm{Si}(001)$.

[16] In the case of the TB-T3 method, $e_{b}$ is the tight-binding 
cohesive energy, which was chosen as the common scale for expressing the total energy.

[17] V.I. Marchenko and A.Y. Parshin, Sov. Phys. JETP 52, 129 (1980).

[18] $\gamma_{114}^{0}=\gamma_{114} / \cos \theta_{0}$ (see Fig. 1), is the projected surface energy of $\mathrm{Si}(114)$.

[19] V.I. Marchenko, Sov. Phys. JETP 54, 605 (1981).

[20] In Table II we have included only the results for cases that satisfy $U_{1} / G_{1}<10$. Beyond this range, the theory developed in [7] shows that the periodicity of the bunch structures exceeds the experimental observations (100$1000 \AA$ ) [1, 2] by nearly 2 orders of magnitude.

[21] This result is consistent with the fact that $U_{n}$ is closely related to the edge energy [7], and should not depend on the number of steps within the bunch.

[22] M. Takeguchi et al., Surf. Sci. 482, 1385 (2002); Surf. Interf. Anal. 30, 288 (2000).

[23] D.J. Chadi, Phys. Rev. Lett. 59, 1691 (1987). 